\title{
The Smooth Curvature Model: An Efficient Representation of Euler-Bernoulli Flexures as Robot Joints
}

\author{
Lael U. Odhner, Member, IEEE, and Aaron M. Dollar, Member, IEEE
}

\begin{abstract}
This paper presents a new method to produce computationally efficient models of robots that have planar elastic flexure joints. An accurate, low-dimensional model of large deformation bending is important to precisely describe the configuration of a flexure-jointed manipulator. The new model is based on the assumption that the curvature of a beam in bending is smooth and, thus, can be approximated by low-order polynomials. This produces a description of flexure motion that can be used as a joint model when expressed as a homogeneous transformation between rigid links-essentially a "drop in" replacement for traditional joint models such as screw coordinates and Denavit-Hartenberg conventions. Derivatives of the joint kinematics such as Jacobians and Hessians are accurate and easy to compute. We will show that with only three parameters, this model faithfully reproduces the elastic deformation of a flexure hinge predicted by the continuum model, even for large angles, without requiring numerical integration or many finite elements. The model can also be used to accurately compute the compliance and compressive buckling load of the flexure, as predicted by the continuum model.
\end{abstract}

Index Terms-Beam bending, flexible, flexures, joints, robot.

\section{INTRODUCTION}

$\mathbf{M}$ ANY robots use joints which consist of highly flexible elastic members, which are referred to as flexures. These features are commonly used to allow motion in monolithic structures via Euler-Bernoulli bending, and have been used as joints in a number of different robotic mechanisms, including compliant hands [1], [2], legged robots [3], and mesoscale flyers [4]. The benefits of flexure-based joints include having no sliding parts (and therefore no friction or stick-slip effects), no backlash, and the ability to compliantly deform in response to unplanned collisions, making them ideal for robots that must operate in unstructured environments [5]. Another major benefit of flexures is the simplicity and potential lower cost compared with standard revolute joints, which require bearings for smooth, accurate

Manuscript received August 1, 2011; revised February 1, 2012; accepted March 28, 2012. Date of publication May 15, 2012; date of current version August 2, 2012. This paper was recommended for publication by Associate Editor A. Albu-Schäffer and Editor B. J. Nelson upon evaluation of the reviewers' comments. Preliminary versions of this paper were presented at the 2010 ASME Mechanisms and Robotics Conference (part of the IDETC conferences), as well as the 2010 Robotics: Science and Systems Conference.

The authors are with the Department of Mechanical Engineering and Materials Science, Yale University, New Hayen, CT 06511 USA (e-mail: lael.odhner@yale.edu; aaron.dollar@yale.edu).

Color versions of one or more of the figures in this paper are available online at http://ieeexplore.ieee.org.

Digital Object Identifier 10.1109/TRO.2012.2193232
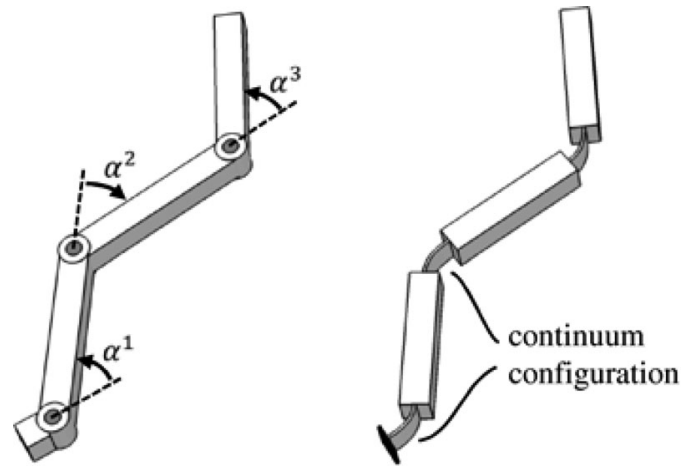

Fig. 1. Comparison of traditional pin joints (left) and flexure joints (right).

motion. As robots become more common as commercial products, flexures are likely to be used with increasing frequency due to their compatibility with inexpensive polymeric fabrication processes such as multishot injection molding [6] and shape deposition manufacturing [7].

One drawback to flexures as robot joints is the complex mechanical behavior that they exhibit compared with pin joints or prismatic joints. A typical open-chain manipulator can be represented by a vector of joint parameters, i.e., $\alpha^{1}, \alpha^{2}, \alpha^{3} \ldots \in \mathbb{R}^{1}$, as illustrated in Fig. 1. The Euclidean transformations between each rigid link of the robot, i.e., $\mathbf{G}^{1}, \mathbf{G}^{2}, \mathbf{G}^{3} \ldots \in \mathrm{SE}(3)$, are functions of these parameters. The partial derivatives of these transformations form the kinematic Jacobians and Hessians used for analysis and control. Flexure-jointed robots are difficult to analyze in an analogous fashion because it is difficult to find a low-dimensional set of parameters accurately describing beam bending, a continuum process. A flexure joint model should satisfy four functional requirements in order to provide both accurate results and be computationally tractable for real-time, closed-loop control.

1) Minimality: The geometric relationship between two links $\mathbf{G}(\boldsymbol{\alpha})$ should be described by a vector $\boldsymbol{\alpha} \in \mathbb{R}^{N}$ having dimensions no larger than the number of degrees of freedom of a single link, i.e., three in the plane and six in space.

2) Energetic fidelity: Any internal energy $U(\boldsymbol{\alpha})$ which is associated with the joint configuration $\alpha$ must be accurately predicted. The minimum energy configuration of the joint should be accurately reproducible in the parameterized representation. 
3) Jacobian fidelity: The first partial derivatives of the joint kinematics $\mathbf{G}(\boldsymbol{\alpha})$ and the internal energy $U(\boldsymbol{\alpha})$ with respect to $\alpha$ should be accurate and easily computed.

4) Hessian fidelity: The second partial derivatives $\mathbf{G}(\boldsymbol{\alpha})$ and $U(\boldsymbol{\alpha})$ with respect to $\boldsymbol{\alpha}$ should also be accurate and easily computed.

There are many extant parametric models of beam bending that can be used to describe joints, but none that currently satisfy all of the aforementioned conditions. One popular class of flexure approximation is the family of pseudorigid body (PRB) models [8]. A PRB model consists of a serial linkage of pin joints and nonlinear springs, fit by regression to the elliptic integral solutions for Euler-Bernoulli beam bending. Because of the quasi-empirical nature of these curve fits, PRB models are only accurate for a limited range of motion and applied force. For instance, the most accurate PRB flexure model, which was reported by $\mathrm{Su}$ in 2009, is incapable of predicting the behavior of a flexure loaded in compression [9].

Another approach with a long history of use within the robotics community is to relate the shape of the flexure, as embodied by the local evolution of the flexure's backbone coordinates, to its elastic and kinetic energy [10], [11]. These continuum descriptions are powerful, but the challenge lies in finding an approximate parameterization of the continuum mechanics that is low dimensional yet accurate. A high-order discretization, such as Bayo's finite element models, obtains accurate results at the cost of using many generalized coordinates to describe a single flexible member [12]. At the other extreme, low-order models, such as constant-curvature segments, are useful for fast computation of difficult inverse kinematics [13], [14]. However, because these models have fewer degrees of freedom than a real flexure, they will not capture finer-grained phenomena such as the elastic forces on a joint or the stiffness of a flexure near buckling.

A logical compromise between simplicity and accuracy is to parameterize bending flexures as a basis of superimposed deformational modes. This technique has been applied to smalldeformation flexible links [15], [16]. The model presented in this paper, i.e., the Smooth Curvature model, is a modal approximation of Euler-Bernoulli bending accurate to large deformations (up to $90^{\circ}$ ), and is consequently capable of accurately representing bending hinges over their entire range of motion. We show that three parameters (consistent with the three degrees of freedom of the planar beam tip) can accurately capture the flexure performance to within $1 \%$ of the exact model, but more parameters can be added to further increase the performance of the model. In contrast with previous joint models, it is fully capable of representing the balance of forces on a flexure joint to a high degree of accuracy through the Jacobian of the joint transformation, and the stiffness properties of the joint through the Hessian of the joint transformation. This paper is an expansion of previous, shorter papers [17], [18] providing a comprehensive introduction to the Smooth Curvature model, as well as new examples, new analysis of the computational cost, and more indepth treatment of higher order behaviors, such as constrained buckling modes.
This paper is structured around the four functional requirements that are outlined previously for parametric joint models. Section II introduces the general problem of parametric approximations to the Euler-Bernoulli equation and demonstrates that the Smooth Curvature model can approximate elastica curves, using a minimal set of parameters, to a high degree of energetic accuracy. Section III then shows that conventional Jacobian analysis can be used to accurately find the equilibrium configuration of a flexure-jointed robot under a variety of loading conditions. Section IV demonstrates the accuracy of the Smooth Curvature model in predicting the stiffness of flexures of arbitrary initial curvature. Additionally, the predicted buckling loads will be shown to be accurate under a wide variety of endpoint constraints.

\section{LOW-DimenSIONAL BEAM MODELS}

\section{A. Introduction}

This section provides an overview of how rigorous, physical parametric models of bending beams can be constructed from the variational form of the Euler-Bernoulli equation. The Smooth Curvature model is introduced as a low-dimensional alternative to more conventional finite element models. The procedure to compute the kinematics and energetics of each joint is shown to be straightforward, and the accuracy of the Smooth Curvature model is compared with those of constant curvature and finite element models by examining the ability of the Smooth Curvature model to approximate the curve of least energy having fixed endpoints and end orientations.

\section{B. Bending Beams as Joints}

Generalizing from the definition given in Section I, we can think of a joint as a function mapping a joint coordinate $\boldsymbol{\alpha} \in \mathbb{R}^{N}$ onto a transformation matrix $\mathbf{G}(\boldsymbol{\alpha}) \in \mathrm{SE}(3)$, representing the geometric relationship between two links. This definition is implicit in any number of widely accepted rigid body modeling notations. For example, Denavit-Hartenberg conventions [19] and screw coordinates [20] are both mappings from $\mathbb{R}^{N}$ onto $S E(3)$ used to parametrically describe links and joints. The configuration vector $\boldsymbol{q}$ of an open-chain, $M$-link robot is the vector that contains all joint parameters:

$$
q=\left[\begin{array}{c}
\boldsymbol{\alpha}^{1} \\
\boldsymbol{\alpha}^{2} \\
\vdots \\
\boldsymbol{\alpha}^{M}
\end{array}\right]
$$

To produce equations of motion in the joint variable space, any internal energy which is associated with a joint's motion, such as elastic energy, should be expressible as a scalar function, i.e., $U(\boldsymbol{\alpha})$. Gravitational and kinetic energy associated with each link can be expressed in terms of the Jacobian of $\mathbf{G}^{1} \ldots \mathbf{G}^{M}$ with respect to $\boldsymbol{q}$. From these, dynamic equations can be assembled in a variety of ways, such as Port-Hamiltonian networks [11], or direct derivation of Newtonian equations of motion by the method of virtual work. Independent of the method chosen, however, it is important that the basis of joint parameters $\alpha$ 


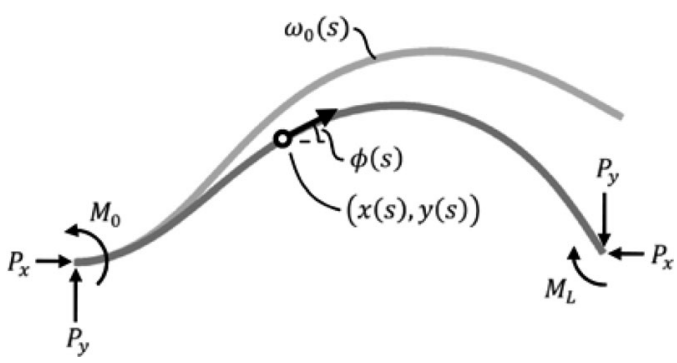

Fig. 2. Terminology used to describe the Euler-Bernoulli equation. The forces and moments at the root of the beam (left) are specified as initial conditions, and the reaction moments at the other end are calculated based on the beam profile.

enables the efficient and accurate computation of $\mathbf{G}, U$, and their partial derivatives. We will now show how a model of the form just described can be obtained for a bending beam, and how the choice of parameterization affects the ease with which a flexure's shape can be computed.

\section{Modeling Large-Deformation Euler-Bernoulli Bending}

We will consider the simplest model of a flexure hinge: the Euler-Bernoulli beam, a thin rod or sheet whose deformation is primarily in bending, rather than tension or shear. The largedeformation Bernoulli model makes the constitutive assumption that the equilibrium rate of rotation along the midline or neutral axis of the beam $\omega(s)$ must be equal to the local bending moment divided by the beam stiffness EI:

$$
\omega(s)=\omega_{0}(s)+\frac{M_{0}+P_{y} x(s)-P_{x} y(s)}{\text { EI }} .
$$

Here, $M_{0}$ is the moment at the root of the beam, $\left(P_{x}, P_{y}\right)$ is the force at the root of the beam, and $\omega_{0}(s)$ is the unforced curvature of the beam, as depicted in Fig. 2. The angle of the tangent vector $\phi(s)$ and the Cartesian profile $(x(s), y(s))$ form a coupled system of ordinary differential equations defined by the local geometry of the neutral axis curve:

$$
\begin{aligned}
\frac{d}{d s} \phi(s) & =\omega_{0}(s)+\frac{M_{0}+P_{y} x(s)-P_{x} y(s)}{\mathrm{EI}} \\
\frac{d}{d s}\left[\begin{array}{l}
x(s) \\
y(s)
\end{array}\right] & =\left[\begin{array}{l}
\cos (\phi(s)) \\
\sin (\phi(s))
\end{array}\right] .
\end{aligned}
$$

Direct methods to solve the Euler-Bernoulli equations typically result in solutions that are based on elliptic integrals [21]. Rather than attempting to shoehorn the parameters that are involved with these integrals into some kind of coordinate basis, the variational form of the Euler-Bernoulli equations can be used, considering the energy of some arbitrary beam curvature $\omega(s)$ and the initial curvature $\omega_{0}(s)$ :

$$
U=\int_{0}^{L} \frac{\mathrm{EI}}{2}\left(\omega(s)-\omega_{0}(s)\right)^{2} d s .
$$

The variational form is powerful because it enables discrete approximations of the continuum problem by substituting the arbitrary configuration $\omega(s)$ with a family of functions $\hat{\omega}(\boldsymbol{\alpha}, s)$, depending on some parameter vector $\boldsymbol{\alpha} \in \mathbb{R}^{N}$. The initial cur-

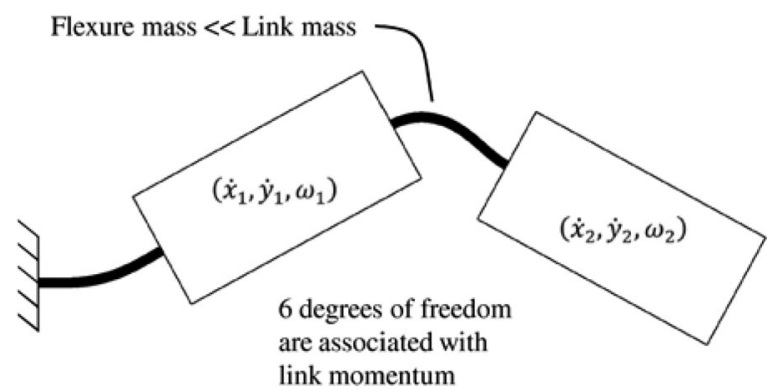

Fig. 3. Two-link planar manipulator will have at most six degrees of freedom associated with the inertia of the links.

vature $\omega_{0}(s)$ is parameterized by a vector $\kappa$ in a fashion identical to the configuration so that $\omega_{0}(s)=\hat{\omega}(\boldsymbol{\kappa}, s)$. The energy of the flexure is then a function of $\alpha$ :

$$
U(\boldsymbol{\alpha})=\int_{0}^{L} \frac{E I}{2}(\hat{\omega}(\boldsymbol{\alpha}, s)-\hat{\omega}(\boldsymbol{\kappa}, s))^{2} d s .
$$

The parameterized, variational form of (3) becomes a decoupled system which can be integrated directly by first solving for $\phi$ and then for $x$ and $y$ :

$$
\begin{aligned}
\frac{d}{d s} \phi(\boldsymbol{\alpha}, s) & =\hat{\omega}(\boldsymbol{\alpha}, s) \\
\frac{d}{d s}\left[\begin{array}{l}
x(\boldsymbol{\alpha}, s) \\
y(\boldsymbol{\alpha}, s)
\end{array}\right] & =\left[\begin{array}{l}
\cos (\phi(\boldsymbol{\alpha}, s)) \\
\sin (\phi(\boldsymbol{\alpha}, s))
\end{array}\right] .
\end{aligned}
$$

At $s=L$, the tip angle $\phi_{\text {tip }}$ and the tip position $\left(x_{\text {tip }}, y_{\text {tip }}\right)$ define the transformation which is associated with this joint:

$$
\mathbf{G}:=\left[\begin{array}{cccc}
\cos \left(\phi_{\text {tip }}\right) & -\sin \left(\phi_{\text {tip }}\right) & 0 & x_{\text {tip }} \\
\sin \left(\phi_{\text {tip }}\right) & \cos \left(\phi_{\text {tip }}\right) & 0 & x_{\text {tip }} \\
0 & 0 & 1 & 0 \\
0 & 0 & 0 & 1
\end{array}\right] .
$$

Equations (5)-(7) form the core of many possible parametric joint models that are based on Euler-Bernoulli bending. The overall procedure - using a discrete basis of assumed deformations to produce a kinematic and energetic approximation of a continuum system-is at the heart of many approximation techniques, including the finite element method. Unlike generic finite element modeling problems, minimizing the number of parameters that are used to describe a robot joint is of paramount importance, not only due to the curse of dimensionality, as discussed by Chirikjian [22]. Flexure joints are much smaller and lighter than the rigid links they connect. Take, for example, the planar system in Fig. 3, consisting of two rigid bodies. A naïve finite element approach might treat a flexure as a chain of many tiny links [12], [21] or constant curvature arcs [10]. However, the inertia of the system will be dominated by the six inertial degrees of freedom of the rigid bodies. The generalized inertia matrix, when constructed for the high-dimensional finite element representation, will be ill-conditioned or singular. Physically, this corresponds to the fact that a joint model having more parameters than the number of degrees of freedom per link will capture not only the dynamic modes that are associated 
with motion of the rigid links but the internal vibrational modes of the flexures as well, which may be on time scales that are orders of magnitude faster than the dynamics of interest.

\section{Choosing a Basis}

To find a low-dimensional basis that sufficiently describes a flexure hinge, a priori knowledge about the equilibrium configurations of Euler-Bernoulli beams must be leveraged in order to find a better, special-purpose basis for joint modeling. Solutions to the large-angle Euler-Bernoulli equation, which are called elastica curves, are smooth-so prototypically smooth, in fact, that bending beams were historically used as drafting tools to reproduce smooth lines on engineering drawings, and serve as the inspiration for modern equivalents in CAD software [23]. It is safe to assume that the curvature profile of a beam in equilibrium will be more efficiently represented by a basis of smooth modal functions, rather than nonsmooth piecewise functions. This idea has been raised in the context of computer graphics, for example, by Horn, who examined the use of clothoid curves as approximations to the computation of natural splines [10], and more recently by Levien [24], in the context of typography. However, these ideas have not yet been applied in the context of robot joints. The Smooth Curvature model approximates the curvature of a bending beam using Legendre polynomials and translated so that they are orthogonal under convolution on the interval from $s=0$ to $s=L$ :

$$
\hat{\omega}(\vec{q}, s)=\frac{\alpha_{1}}{L}+\frac{\alpha_{2}}{L}\left(\frac{2 s}{L}-1\right)+\frac{\alpha_{3}}{L}\left(\frac{6 s^{2}}{L^{2}}-\frac{6 s}{L}+1\right) .
$$

The coefficients $\boldsymbol{\alpha}=\left[\begin{array}{lll}\alpha_{1} & \alpha_{2} & \alpha_{3}\end{array}\right]^{T}$ serve as the generalized coordinates that describe the flexure configuration. A third-order approximation was chosen to stay within the budget of three planar rigid-body degrees of freedom.

The energy function under this Smooth Curvature basis can be integrated analytically from (5). As a happy side effect of this particular orthogonal basis, the bending energy can be written as a sum of squares in terms of $(\boldsymbol{\alpha}-\boldsymbol{\kappa})$ :

$$
U(\vec{q})=\frac{\mathrm{EI}}{2 L}\left(\left(\alpha_{1}-\kappa_{1}\right)^{2}+\frac{\left(\alpha_{2}-\kappa_{2}\right)^{2}}{3}+\frac{\left(\alpha_{3}-\kappa_{3}\right)^{2}}{5}\right) .
$$

Recall that the tip angle $\phi_{\text {tip }}$ and the tip position $\left(x_{\text {tip }}, y_{\text {tip }}\right)$ are needed to define $\mathbf{G}(\boldsymbol{\alpha})$. The tip angle can be analytically integrated, and the choice of basis simplifies the result

$$
\phi(\boldsymbol{\alpha}, s)=\alpha_{1} \frac{s}{L}+\alpha_{2}\left(\frac{s^{2}}{L^{2}}-\frac{s}{L}\right)+\alpha_{3}\left(\frac{2 s^{3}}{L^{3}}-\frac{3 s^{2}}{L^{2}}+\frac{s}{L}\right)
$$

$$
\phi_{\text {tip }}(\boldsymbol{\alpha})=\phi(\boldsymbol{\alpha}, L)=\alpha_{1} \text {. }
$$

Because $\phi(\boldsymbol{\alpha}, s)$ is a cubic polynomial, the definite integrals from (5) defining the tip position are transcendental functions:

$$
\begin{aligned}
& x_{\mathrm{tip}}(\boldsymbol{\alpha})=\int_{0}^{L} \cos (\phi(\boldsymbol{\alpha}, s)) d s \\
& y_{\mathrm{tip}}(\boldsymbol{\alpha})=\int_{0}^{L} \sin (\phi(\boldsymbol{\alpha}, s)) d s .
\end{aligned}
$$

Fortunately, the same smoothness assumptions that motivated the Legendre polynomial basis imply that the integrands in (12) and (13) are smooth functions; therefore, a low-order quadrature integration method such as Gauss-Legendre quadrature should be fast and accurate [25]. The end-to-end tip displacement of the flexure is, therefore, approximately a weighted sum of the integrands from (12) and (13), using partition weights $\Delta_{k}$ and sampled points $s_{k}$ :

$$
\begin{aligned}
& x_{\mathrm{tip}}(\boldsymbol{\alpha})=L \sum_{k=1}^{N} \Delta_{k} \cos \left(\phi\left(\boldsymbol{\alpha}, s_{k}\right)\right) \\
& y_{\mathrm{tip}}(\boldsymbol{\alpha})=L \sum_{k=1}^{N} \Delta_{k} \sin \left(\phi\left(\boldsymbol{\alpha}, s_{k}\right)\right) .
\end{aligned}
$$

The weights and partition points that are used for the GaussLegendre quadrature can be found in Appendix A. Five partition points were found to compute the integral with sufficient accuracy; only ten trigonometric function evaluations are needed to compute the link-to-link transformation matrix for the flexure. Consequently, the burden of computing $\mathbf{G}(\boldsymbol{\alpha})$ is fairly low.

\section{E. Improvements in Model Order}

One implication of the variational Euler-Bernoulli equation is that the energy of bending from (4) will be minimized by the equilibrium configuration of the flexure $\omega^{*}(s)$ for any fixed set of flexure boundary conditions [10]. Consequently, any parameterized configuration $\hat{\omega}\left(\boldsymbol{\alpha}^{*}, s\right)$ will have a bending energy that is equal to or greater than the bending energy of the true flexure configuration having the same fixed endpoints. The deviation of the approximate solution from the true solution can be captured by the ratio of the two energies, written here as the error function $\eta:$

$$
\eta=\frac{U\left(\boldsymbol{\alpha}^{*}\right)}{U^{*}}-1
$$

Here, $U^{*}$ is the minimum energy that corresponds to the actual equilibrium configuration $\omega^{*}(s)$, and $U\left(\boldsymbol{\alpha}^{*}\right)$ is the energy that corresponds to the approximated equilibrium configuration $\boldsymbol{\alpha}^{*}$. This error function is nonnegative and zero only when $\hat{\omega}\left(\boldsymbol{\alpha}^{*}, s\right)=\omega^{*}(s)$. It is a natural choice of standard to compare different approximations.

To demonstrate the power of choosing the correct basis functions for representing flexure curvature, the Smooth Curvature model was compared with a finite element model which was made up of constant curvature segments, performing the task to find minimum energy curves between two points in the plane. The goal was to find the model order of the finite element model having comparable energetic accuracy. The fixed boundary conditions that are chosen for comparison are shown in Fig. 4. The value of $U^{*}$ was computed by explicitly solving the EulerBernoulli equation, iteratively shooting to satisfy the boundary conditions.

Because the Smooth Curvature model uses only three parameters, no actual minimization process was needed to find the flexure configuration satisfying the three boundary conditions of the flexure. Instead, (11), (14), and (15) were solved numerically: 


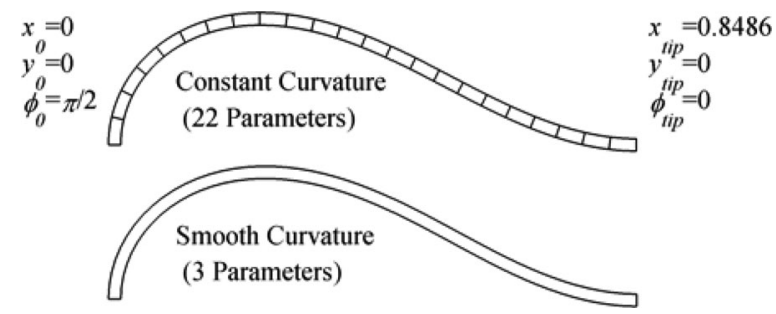

Fig. 4. Comparison of the minimum-energy curves connecting two points, assuming a basis of constant curvature finite elements (top) and the smooth curvature basis (bottom). Twenty-two finite elements are needed to achieve the energetic accuracy of three Legendre polynomials.

a process made simpler by the fact that (11) independently constrains the value of $\alpha_{1}$ to $\phi_{\text {tip }}$. The tip position was solved for $x_{\text {tip }}$ and $y_{\text {tip }}$ in terms of $\alpha_{2}$ and $\alpha_{3}$ alone. The finite element model was solved as a constrained energy minimization problem using MATLAB.

The energy error of the Smooth Curvature solution was found to be 0.0016 . To match this error, the number of elements that are used in the finite element model was iteratively increased until the value of $\eta$ for its solution was below the Smooth Curvature model's solution. This required 22 elements and yielded an error of $\eta=0.0013$.

\section{F. Discussion}

The approximated equilibrium profiles are shown in Fig. 5, and lend some insight as to why the Smooth Curvature model requires many fewer parameters to represent the equilibrium curvature of a flexure. First, the plot graphically illustrates that constant curvature finite elements produce, in effect, a zeroorder hold approximation to the true curvature function. This description will not be sparse-there is no reason a priori to assume that the curvature anywhere is more likely to be zero, and thus, no way to directly reduce the amount of information needed to reproduce the curvature. In addition, a zero-order hold approximation is canonically nonsmooth. It will introduce high-frequency errors that are mitigated only by the fact that the curvature is successively integrated to produce the flexure profile. In contrast, the quadratic polynomial approximation does a much better job of representing the equilibrium curvature, as it is smooth in all its derivatives, and is also sparse in the sense that high-order terms can be reasonably expected to matter less in describing the overall shape of the flexure.

\section{G. Summary}

In this section, we have shown that the Smooth Curvature model is a low-order alternative to conventional finite element models of Euler-Bernoulli bending members. The three variational parameters that are used to represent the internal configuration of the flexure joint can be used as joint parameters in a robot model, and the cost to compute the bending energy and forward kinematics of the model is realistic. In the next sections, we will see that the higher order behaviors of this model, that is, phenomena dependent on first and second derivatives of the

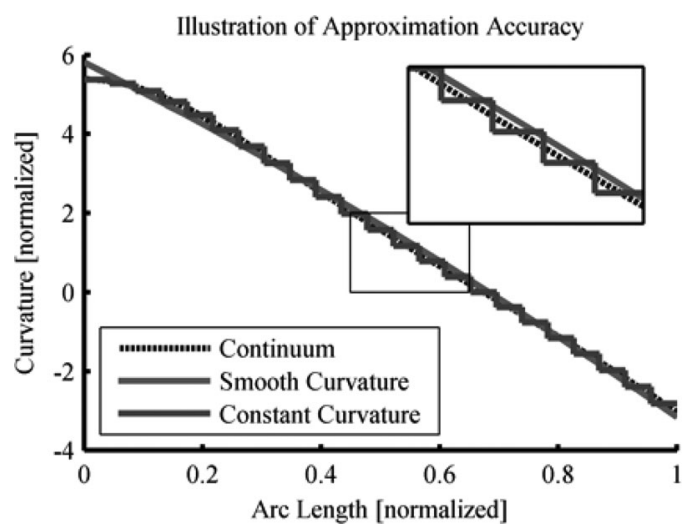

Fig. 5. Smooth Curvature model is a sparse approximating basis for the equilibrium shapes of a bending beam, meaning that few nonzero terms are needed to reproduce most of the beam's shape. In contrast, the piecewise constant curvature model does not provide a sparse representation; thus, many more terms are needed.

kinematics, are also accurate. Thus, notions such as the Jacobian or Hessian of a point with respect to the joint variables can be cleanly transferred from more traditional joints to flexure joints.

\section{JACOBIAN ANALYSIS OF FLEXURE JOINTS}

\section{A. Introduction}

In addition to computing the pose of a robot from its joint parameters, it is also important that a joint model accurately represents the force and instantaneous motion of a robot from the generalized velocities of the joint parameters $\dot{\boldsymbol{q}}$. The dual space of generalized forces $\tau$ should describe all the conditions for the equilibrium of the robot. For example, if a force $f$ at some point $\boldsymbol{w}$ is applied to the robot, then the deformed configuration of the system can be found using the Jacobian of that point's coordinates and the gradient of the summed internal energy of the robot $V$ :

$$
\boldsymbol{\tau}=-\nabla_{\boldsymbol{q}} V+\mathcal{J}_{w}^{T} \boldsymbol{f}=\mathbf{0}
$$

The generalized force balance equation is only realistic if the energy gradient and kinematic Jacobian faithfully represent all of the motions that the robot is capable of making. It is also important that these quantities are easy to compute; therefore, a force balance of the kind shown in (17) can be integrated into algorithms for analysis, estimation, and control. This section demonstrates the ability of the Smooth Curvature model to predict deformation under load, both in terms of the positional and angular errors, entirely within the framework of joint parameters as generalized coordinates.

\section{B. Partial Derivatives of the Smooth Curvature Model}

The partial derivatives of the energy $U(\boldsymbol{\alpha})$ and the link-tolink transformation matrix $\mathbf{G}(\boldsymbol{\alpha})$ under the smooth curvature parameterization previously introduced are straightforward to obtain. The partial derivatives of $U(\boldsymbol{\alpha})$ from (9) can be written 


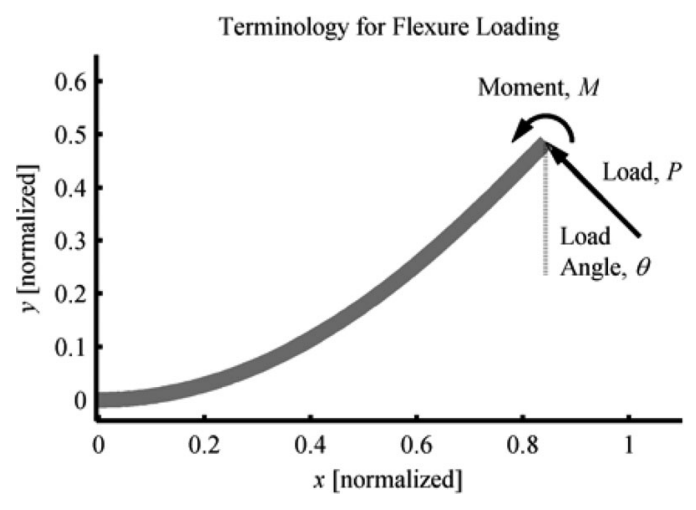

Fig. 6. Description of the parameterized end load applied to the flexure in each test case.

as a linear, spring-like matrix:

$$
\nabla_{\alpha} U=\frac{\mathrm{EI}}{L}\left(\begin{array}{ccc}
1 & 0 & 0 \\
0 & 1 / 3 & 0 \\
0 & 0 & 1 / 5
\end{array}\right)\left[\begin{array}{l}
\alpha_{1} \\
\alpha_{2} \\
\alpha_{3}
\end{array}\right] .
$$

The partial derivatives of $\mathbf{G}(\boldsymbol{\alpha})$ can be found by taking the partial derivatives of the flexure backbone coordinates from (6), and then integrating using the Gauss-Legendre quadrature. Because partial differentiation with respect to $\alpha_{i}$ and integration over $s$ on a fixed interval are commutative operations, this is equivalent to taking the partial derivatives of (11), (14), and (15):

$$
\begin{aligned}
& \frac{\partial \phi_{\text {tip }}}{\partial \alpha_{i}}= \begin{cases}1, & i=1 \\
0, & i \neq 1\end{cases} \\
& \frac{\partial x_{\text {tip }}}{\partial \alpha_{i}}=-L \sum_{k=1}^{N}\left(\Delta_{k} \frac{\partial \phi\left(s_{k}\right)}{\partial \alpha_{i}}\right) \sin \left(\phi\left(\boldsymbol{\alpha}, s_{k}\right)\right) \\
& \frac{\partial y_{\text {tip }}}{\partial \alpha_{i}}=L \sum_{k=1}^{N}\left(\Delta_{k} \frac{\partial \phi\left(s_{k}\right)}{\partial \alpha_{i}}\right) \cos \left(\phi\left(\boldsymbol{\alpha}, s_{k}\right)\right) .
\end{aligned}
$$

Many of the mathematical operations that are needed to compute these partial derivatives, including all of the ten trigonometric function evaluations, can be recycled from (14) and (15). The partial derivatives of $\phi$ at each partition point are independent of $\boldsymbol{\alpha}$; Appendix A includes an example program to calculate these values.

\section{Verifying the Accuracy of Jacobian Analysis}

The accuracy of (19)-(21) can be ascertained by considering a simple, dimensionless test case, such as the one shown in Fig. 6. A clamped beam is loaded at the free end with an arbitrary moment $M$ and force $P$ exerted at an angle $\theta$ so that $P_{x}=$ $-P \sin \theta$ and $P_{y}=P \cos \theta$. Using the Jacobian of the flexure transformation, the end load can be projected onto generalized coordinates:

$$
\boldsymbol{\tau}=\nabla_{\alpha} U-\nabla_{\alpha}\left(\phi_{\mathrm{tip}} M\right)-\nabla_{\alpha}\left(x_{\mathrm{tip}} P_{x}\right)-\nabla_{\alpha}\left(y_{\mathrm{tip}} P_{y}\right) .
$$

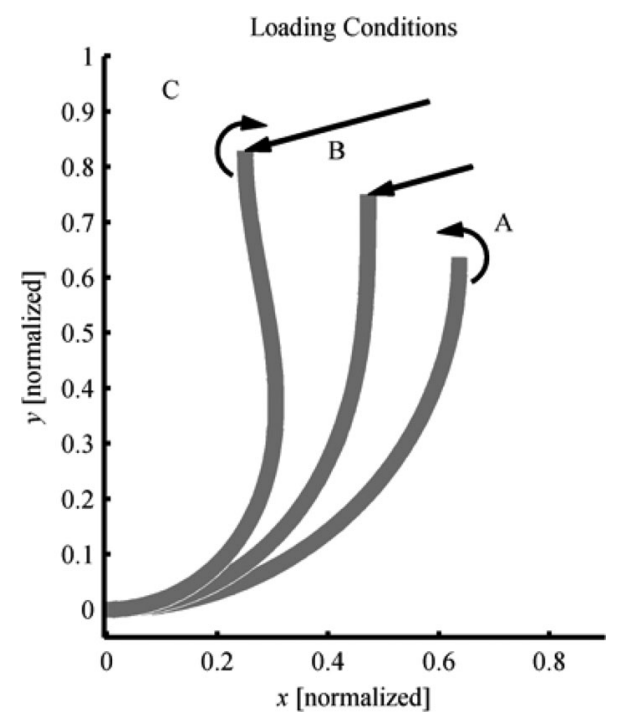

Fig. 7. Three loading cases considered for this study. (A) pure moment, (B) pure force, and (C) pure force opposed by a moment.

When set equal to zero, this system of nonlinear equations can be solved to find the deformed joint configuration. To find the prediction error of the Smooth Curvature model, the EulerBernoulli equations from (3) were solved as a boundary value problem by shooting, that is, iteratively varying the moment at the root of the beam until the boundary conditions at both ends of the beam are satisfied. The dimensionless form of the beam bending equations was used so that the flexure stiffness $E I$ and the flexure length $L$ were normalized to 1 . Rather than performing an exhaustive search over the space of loading conditions for the flexure, an extreme case, in which the deformed tip angle $\phi_{\text {tip }}$ is equal to $90^{\circ}$, was chosen for comparison. This is a generalization of the rectangular elastica problem posed by Bernoulli, the problem of finding the shape of a cantilevered beam bent at a right angle by a perpendicular force at the tip [26]. Three force-moment combinations were chosen to compare the two models. The test loading conditions, which are labeled A, B, and $\mathrm{C}$, are shown in Fig. 7. In case A, a pure bending moment was applied, sufficient to bend the flexure to an angle of $90^{\circ}$. In case $\mathrm{B}$, a pure force of a magnitude sufficient to bend the flexure to $90^{\circ}$ was applied. Case $\mathrm{C}$ consisted of a moment equal and opposite to the moment applied in combination 1, counteracted by a force so that the flexure tip angle remained at $90^{\circ}$.

The positional error of the flexure tip was found as the norm of the vector from the predicted flexure tip to the tip of the numerically computed elastica curve. This is plotted in nondimensional form, meaning that the error is given as a fraction of the flexure length (see Fig. 8) with the angular error. These plots show a number of significant results. First, the errors in case A (pure moment loading) were very small. The exact shape one would expect for a beam having a constant bending moment is a constant curvature arc, a shape that can be exactly reproduced with the Smooth Curvature model if $q_{2}=q_{3}=0$. Thus, the error is correspondingly small. The errors observed in loading cases B and C indicate that the three-parameter Smooth 

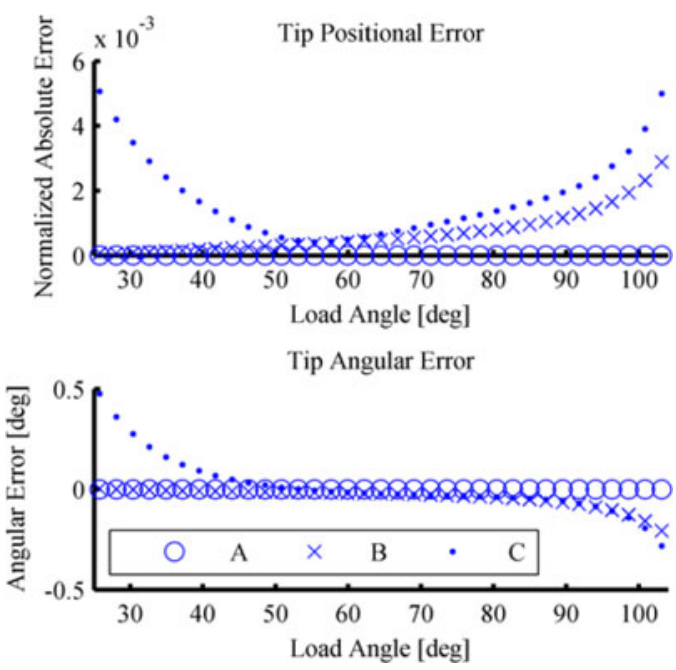

Fig. 8. Positional errors (top) and angular errors (bottom) of the three test cases.

Curvature model is accurate even when the flexure is loaded by an opposed force and moment. Most importantly, in every case, the results from the Smooth Curvature model were within a positional accuracy of $1 \%$ of the beam length, and an angular accuracy of $1^{\circ}$.

\section{Example: A Tendon-Driven Flexure Joint}

The value in having a parametric joint model is to move beyond the simple force-displacement model of flexure behavior. Ideally, the Jacobian formulation can be used in abstract to analyze the behavior of a whole mechanism. A practical problem typically occurring in tendon-driven flexure mechanisms such as the SDM Hand [1] is to predict the rotation of a single link as a function of tendon force, as illustrated in Fig. 9. This would be useful in order to determine the size of actuator required to drive a joint or to predict the link position and orientation with only a measure of tendon tension. As always, each point on the robot can be represented as a tree-like composition of rigid body transformations, shown as arrows in the figure. The tendon, which is stretched across the joint, is defined relative to the endpoints of the flexure by constant transformations $\mathbf{T}_{1}$ and $\mathbf{T}_{2}$. The joint transformation $\mathbf{G}$ is defined by the $3 \times 1$ configuration vector $\boldsymbol{\alpha}$. This problem can be solved in a manner similar to the aforesaid elastica examples. First, an expression for the energy in the system is needed, assuming that the tension force $f$ is constant. The work done on the tendon is equal to the tendon force multiplied by the length of the tendon, $|\Delta x|$ :

$$
\left[\begin{array}{cc}
\mathbf{R}(\boldsymbol{\alpha}) & \Delta x(\boldsymbol{\alpha}) \\
0 & 1
\end{array}\right]=\mathbf{T}_{1}^{-1} \mathbf{G}(\boldsymbol{\alpha}) \mathbf{T}_{2}
$$

The net energy is the sum of external tendon work and internal elastic energy:

$$
E(\boldsymbol{\alpha})=\frac{\mathrm{EI}}{2 L}\left(\alpha_{1}^{2}+\frac{\alpha_{2}^{2}}{3}+\frac{\alpha_{3}^{2}}{5}\right)-f|\Delta x(\boldsymbol{\alpha})| .
$$

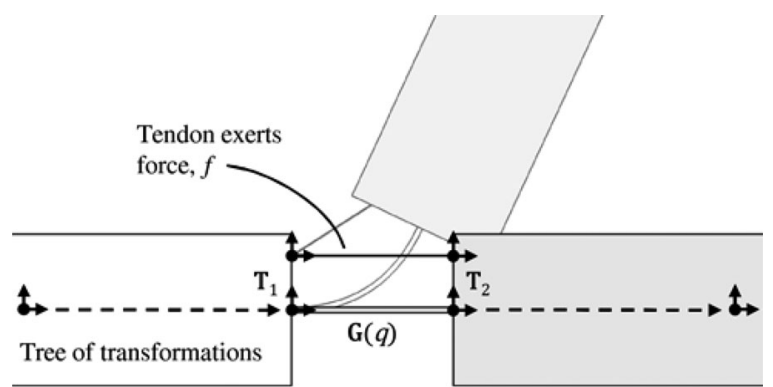

Fig. 9. Deformation of a tendon-driven flexure joint is illustrated previously. The tree of homogeneous transformations along the manipulator defines the length of the tendon and can be used to compute the equilibrium configuration for any tendon load.

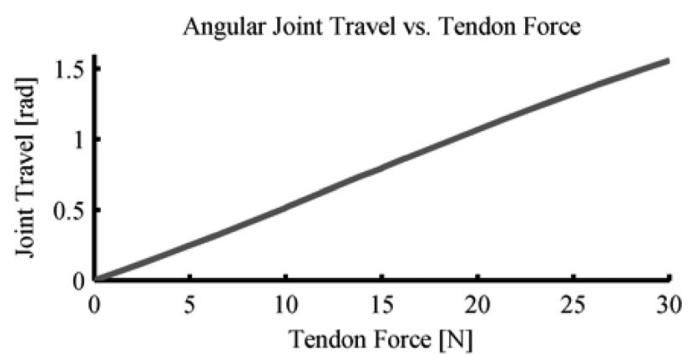

Fig. 10. Predicted joint rotation as a function of tendon force.

The configuration minimizing this energy function will be the equilibrium configuration for the finger. Suppose that a steel flexure that is $0.2 \mathrm{~mm}$ thick, $10 \mathrm{~mm}$ wide, and $L=15 \mathrm{~mm}$ long is used, with a tendon connected at $5-\mathrm{mm}$ offsets from each end of the flexure. The bending stiffness of the thin flexure is given by these dimensions, the elastic modulus of steel (200 GPa), and Poisson's ratio (0.29):

$$
\begin{aligned}
\mathrm{EI} & =\frac{2 \times 10^{11}}{1-0.29^{2}} \frac{0.01 \times 0.0002^{3}}{12} \\
& =1.46 \times 10^{-3} \frac{\mathrm{Nm}}{\mathrm{rad}} .
\end{aligned}
$$

The transformations between the ends of the flexure and the tendons are both given by the homogeneous transformation matrix

$$
T_{1}=T_{2}=\left[\begin{array}{cccc}
1 & 0 & 0 & 0 \\
0 & 1 & 0 & 0.005 \\
0 & 0 & 1 & 0 \\
0 & 0 & 0 & 1
\end{array}\right] .
$$

Differentiating (24) with respect to the joint parameters, generalized force balance equations were solved numerically to obtain the equilibrium configuration of the joint as tendon force was increased. The relationship between tendon force and angular rotation is plotted in Fig. 10, predicting approximately $28 \mathrm{~N}$ as the tendon tension required to rotate the joint to a right angle.

\section{E. Summary}

For a dimensionless flexure hinge, the Jacobian of the Smooth Curvature model captures conditions for equilibrium 
of a flexure-based robot and accurately reproduces the robot's shape as a function of arbitrary applied loads. This is important because of the abstraction it affords. Because no special-purpose solver is needed to account for joint elasticity, it would be possible to model the deformation under load of a robot having a mixture of pin joints and flexure joints. Other properties that depend on the Jacobians of a robot's shape with respect to joint variables, such as the generalized inertia matrix, should be similarly accurate and computationally inexpensive.

\section{STIFFNESS AND BUCKLING}

\section{A. Introduction}

Although much of the literature surrounding flexure joints centers on predicting deflection under load, second-order effects, such as stiffness and buckling, are critical to the accurate modeling and control of a robot. For example, it is well known that the stiffness of a manipulator can play a significant role in grasp stability [27], [28]. Buckling is also a major concern introduced by the use of bending beam elements as joints. The Smooth Curvature model treats stiffness and buckling in the conventional framework of a joint parameter stiffness matrix and produces accurate results despite the relatively constrained space of flexure configurations used by the model.

As in the previous sections, we will proceed by introducing the basic mechanics of second-order effects in flexure joints, and follow this with a comparison between the continuum mechanics of Euler-Bernoulli bending and the results of the smooth curvature model. Two modeling tasks will be demonstrated. First, we will compare the Euler-Bernoulli model's prediction of flexure compliance under a small tip load with the results obtained via Jacobian and Hessian analysis of the smooth curvature model. The algebraic result that is obtained for the Smooth Curvature model is considered in the context of compliance programming. Second, we will show that the determinant of a flexure's stiffness matrix can be used to predict the Euler buckling loads of a flexure under a wide variety of end constraints. Most importantly, both of these tasks can be accomplished in the framework of the generalized stiffness matrix, with no need for ad hoc augmentation of the basic rigid body modeling framework.

\section{B. Generalized Stiffness}

The generalized stiffness matrix $\mathbf{K}$ of a holonomically constrained manipulator can be thought of as the Hessian of the energy associated with a manipulator's joint coordinates. For example, consider the simple manipulator shown in Fig. 11, having generalized coordinates $\boldsymbol{q}$, elastic energy $V$, and a force $\boldsymbol{f}$ exerted at $\boldsymbol{w}$. The generalized stiffness is the summed Hessian of both the internal and external work:

$$
K_{i j}(\boldsymbol{q})=\frac{\partial^{2} V}{\partial q_{i} \partial q_{j}}+\sum_{k} \frac{\partial^{2} w_{k}}{\partial q_{i} \partial q_{j}} f_{k} .
$$

From this, one can see that the second derivatives of both the internal energy and the link kinematics are needed to predict generalized stiffness. For the smooth curvature model, the problem of finding second partial derivatives of energetic and

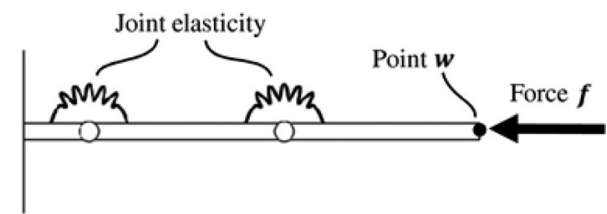

Fig. 11. Both elastic elements and external forces affect the generalized stiffness matrix of a robot, resulting in complex behaviors such as buckling.

kinematic expressions is relatively straightforward. The second partial derivatives of energy form a constant diagonal matrix, as if a set of springs were attached to each parameter:

$$
\nabla \nabla_{\alpha} U=\frac{\mathrm{EI}}{L}\left[\begin{array}{ccc}
1 & 0 & 0 \\
0 & 0 / 3 & 0 \\
0 & 0 & 1 / 5
\end{array}\right]
$$

The Hessians of the kinematic equations can be derived directly from (16)-(18) and simplify drastically because the second derivatives of $\phi(\alpha, s)$ with respect to any joint parameter are zero:

$$
\begin{aligned}
\frac{\partial^{2} \phi_{\mathrm{tip}}}{\partial \alpha_{i} \partial \alpha_{j}} & =0 \\
\frac{\partial^{2} x_{\mathrm{tip}}}{\partial \alpha_{i} \partial \alpha_{j}} & =-L \sum_{k=1}^{N}\left(\Delta_{k} \frac{\partial \phi\left(s_{k}\right)}{\partial \alpha_{i}} \frac{\partial \phi\left(s_{k}\right)}{\partial \alpha_{j}}\right) \cos \left(\phi\left(s_{k}\right)\right) \\
\frac{\partial^{2} y_{\mathrm{tip}}}{\partial \alpha_{i} \partial \alpha_{j}} & =-L \sum_{k=1}^{N}\left(\Delta_{k} \frac{\partial \phi\left(s_{k}\right)}{\partial \alpha_{i}} \frac{\partial \phi\left(s_{k}\right)}{\partial \alpha_{j}}\right) \sin \left(\phi\left(s_{k}\right)\right) .
\end{aligned}
$$

The only difference between the computation of the forward kinematics, the Jacobians, and the Hessians is the additional partial derivatives of $\phi$. Because these are functions of $s$ alone, they can be lumped into weighting coefficients computed $a$ priori for summation of the trigonometric terms common to all three. Computation of stiffness for a single joint requires only the same six trigonometric function evaluations made in (14) and (15) as well as (17) and (18), making the burden of computation fairly low. The stiffness matrix of a single flexure can then be constructed from (28)-(31):

$$
\mathbf{K}=\nabla \nabla_{\alpha} U-\left(\nabla \nabla_{\alpha} x_{\text {tip }}\right) P_{x}-\left(\nabla \nabla_{\alpha} y_{\text {tip }}\right) P_{y} .
$$

Because the Hessian of the tip angle with respect to the generalized coordinates is identically zero, moments on the tip will not directly affect the generalized stiffness matrix.

\section{Small-Force Stiffness Analysis}

One problem that has been previously posed for flexurejointed, rigid-body robots is the prediction of tip compliance for a flexure under small end loads, given some undeformed flexure shape. This problem has appeared in the context of compliance programming for flexure hinges [29], that is, choosing some initial unloaded shape for a flexure joint to control its end-to-end compliance, which can be written as a $3 \times 3$ matrix 
C:

$$
\left[\begin{array}{l}
\Delta \phi \\
\Delta x \\
\Delta y
\end{array}\right] \approx \mathbf{C}\left[\begin{array}{c}
M \\
-P \sin \theta \\
P \cos \theta
\end{array}\right]
$$

Here the tip load, which is assumed to be small in magnitude relative to the Euler buckling load of the flexure, is defined by $M, P$, and $\theta$ (see Fig. 6). In the continuum case, one might find $\mathbf{C}$ from an initial curvature profile $\omega_{0}(s)$ by expressing the evolution of compliance along the beam as an ordinary differential equation:

$$
\begin{aligned}
\frac{d \mathbf{C}}{d s} & =\mathbf{A C}+\mathbf{C A}^{T}+\mathbf{B} \\
\mathbf{A} & =\left[\begin{array}{ccc}
0 & 0 & 0 \\
-\sin (\phi(s)) & 0 & 0 \\
\cos (\phi(s)) & 0 & 0
\end{array}\right], \quad \mathbf{B}=\left[\begin{array}{ccc}
1 / \mathrm{EI} & 0 & 0 \\
0 & 0 & 0 \\
0 & 0 & 0
\end{array}\right] .
\end{aligned}
$$

The matrix A represents the growing moment arm of the flexure; $\mathbf{B}$ represents the incremental rotational compliance of the beam. The derivation of (34) is straightforward and has been omitted for brevity. A more general treatment of compliance in thin beams can be found in [29]. Rather than solving the continuum problem, one could couch this problem in terms of joint stiffness matrices. Using the Smooth Curvature model, the equivalent problem is to find the end-to-end stiffness of a joint having some unloaded configuration $\kappa$. When the tip loads are small in comparison with the elastic term, $\nabla \nabla_{\alpha} U$, the joint stiffness matrix from (32) will be constant:

$$
\mathbf{K}=\nabla \nabla_{\alpha} U=\frac{E I}{L}\left[\begin{array}{ccc}
1 & 0 & 0 \\
0 & 1 / 3 & 0 \\
0 & 0 & 1 / 5
\end{array}\right] .
$$

The tip Jacobian $\mathcal{J}(\boldsymbol{\kappa})$ in the unloaded configuration can be computed as in (16)-(19) to find tip stiffness from the generalized stiffness in the absence of external loads [30]:

$$
\mathbf{C}_{s c}(\kappa)=\mathcal{J}(\boldsymbol{\kappa}) \mathbf{K}^{-1} \mathcal{J}(\boldsymbol{\kappa})^{T} .
$$

Thus, the problem of compliance programming with flexures could be cast into the framework of choosing $\kappa$ to determine $\mathbf{C}$, rather than solving an initial value problem from (34).

A comparison between the smooth curvature model and the integral method of (34) was obtained by computing the tip compliance of a dimensionless flexure whose initial constant curvature $\omega_{0}(s)$ varied from 0 to $\pi$, as depicted in Fig. 12. The integral method was solved using the second-order Runge-Kutta integration with a fixed interval width of $\Delta s=0.0001$. The resulting compliance matrices were extremely close to the values that are predicted by the Smooth Curvature model having the corresponding value of $\kappa$, as shown in Fig. 13 The elementwise infinity norm of the error (that is, the absolute magnitude of the largest error element) is plotted as a function of the flexure curvature. In all cases, the maximum error is $7.33 \times 10^{-4}$. To give a sense of scale, the compliance matrix predicted by (34)

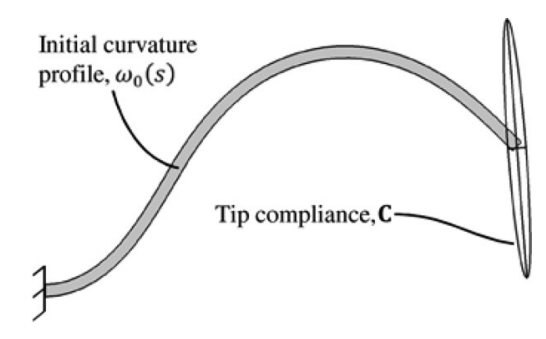

Fig. 12. Compliance ellipse of a beam varies based on the initial curvature profile.

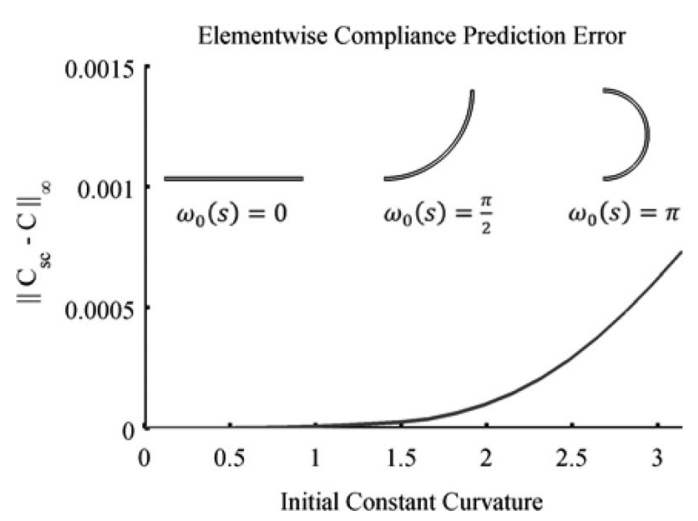

Fig. 13. Maximum elementwise error in the compliance ellipse of a flexure is shown for flexures of varying constant curvature. The maximum elementwise error occurs in the case of largest initial curvature and is still small compared with the magnitude of the elements in the compliance matrix.

for the case when $\omega_{0}(s)=\pi$ is equal to

$$
\mathbf{C}=\left[\begin{array}{ccc}
1.0000 & -0.3183 & -0.2026 \\
-0.3183 & 0.1520 & 0.0645 \\
-0.2026 & 0.0645 & 0.0507
\end{array}\right]
$$

These brief results confirm that the Smooth Curvature model is a viable method to predict the unloaded planar compliance of flexure hinges and, by extension, that it is a possible substitute for more complex integral methods to solve compliance programming and other similar design problems.

\section{Large-Load Analysis and Euler Buckling}

The external loads on a robot are frequently large enough to result in nonnegligible changes in the stiffness of the robot's structure. For example, recall the example from Section III$\mathrm{D}$, a single joint actuated by a tendon. The tendon exerts a compression force on the flexure joint that will tend to make the flexure buckle, i.e., exhibit zero lateral stiffness. The flexure's buckling load $P_{\text {crit }}$ is given by Euler's formula:

$$
P_{\text {crit }}=\frac{\pi^{2}}{4} \frac{\mathrm{EI}}{L^{2}}=2.4674 \frac{\mathrm{EI}}{L^{2}}=15.76 \mathrm{~N} .
$$

In other words, the buckling load of the flexure hinge is right in the middle of the tendon force range depicted in Fig 10. 
This does not to imply that the flexure is unstable; the flexure deforms from its straightened configuration before the buckling load is reached. However, it does indicate that the stiffness terms due to tip loading are large enough to be nonnegligible. To demonstrate the Smooth Curvature model's ability to predict large-load stiffness, we will examine the buckling load of flexure hinges under a variety of end constraints. Buckling is a good proxy case to understand a model's ability to predict large-load stiffness. It could be interpreted as the special case in which the elastic stiffness of a structure is of the same magnitude as the destabilizing effect due to an external load. It is also a case for which gold standard analytical models exist.

The stiffness matrix of a straightened flexure loaded in compression, from (32), would have one term corresponding to the elastic energy in the flexure and one due to the Hessian of the flexure tip's $x$ coordinate computed using (30):

$$
\mathbf{K}=\frac{\mathrm{EI}}{L}\left[\begin{array}{ccc}
1 & 0 & 0 \\
0 & \frac{1}{3} & 0 \\
0 & 0 & \frac{1}{5}
\end{array}\right]+\mathrm{LP}_{\text {crit }}\left[\begin{array}{ccc}
-\frac{1}{3} & \frac{1}{12} & \frac{1}{60} \\
\frac{1}{12} & -\frac{1}{30} & 0 \\
\frac{1}{60} & 0 & -\frac{1}{210}
\end{array}\right]
$$

The numerical errors in the tip Hessian are extremely small in the straightened configuration; therefore, the exact fractions are shown; however, the numerical results shown were obtained using the numerically computed Hessian. The load at which the flexure buckles can be found by setting the determinant of (39) is equal to zero:

$$
\left|\begin{array}{ccc}
\frac{\mathrm{EI}}{L^{2} P_{\text {crit }}}-\frac{1}{3} & \frac{1}{12} & \frac{1}{60} \\
\frac{1}{12} & \frac{\mathrm{EI}}{3 L^{2} P_{\text {crit }}}-\frac{1}{30} & 0 \\
\frac{1}{60} & 0 & \frac{\mathrm{EI}}{5 L^{2} P_{\text {crit }}}-\frac{1}{210}
\end{array}\right|=0 .
$$

The smallest value of $P_{\text {crit }}$ satisfying this expression agrees with Euler's result from (38) to within $0.02 \%$.

$$
P_{\text {crit }}=2.4677 \frac{\mathrm{EI}}{L^{2}}
$$

Predicting higher order buckling modes is also important. Manipulators frequently have parallel kinematic chains which impose internal constraints. To examine these cases, several well-known tip constraints were imposed on the Smooth Curvature model which is depicted in Fig. 14. In order to approximate the discrete buckling loads under tip constraints, the stiffness matrix was constrained to consider only the admissible perturbations in parameter space. Thus, a clamped-guided beam,

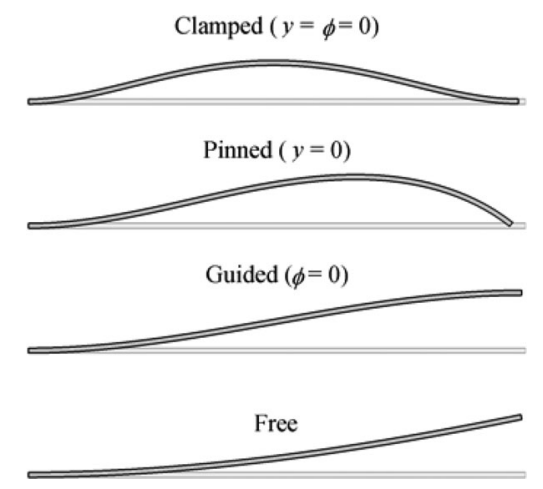

Fig. 14. Buckling modes corresponding to the constrained flexure tip boundary conditions for which buckling loads were predicted are shown.

which cannot rotate at the tip, must hold $\alpha_{1}$ fixed; therefore, the constrained stiffness $\mathbf{K}_{\text {guided }}$ is approximately ${ }^{1}$

$$
\mathbf{K}_{\text {guided }}=\left[\begin{array}{lll}
0 & 1 & 0 \\
0 & 0 & 1
\end{array}\right] \mathbf{K}\left[\begin{array}{ll}
0 & 0 \\
1 & 0 \\
0 & 1
\end{array}\right] .
$$

Similarly, a clamped-clamped beam can neither rotate nor translate at the tip, meaning that the parametric constraint on motion is that $\alpha_{1}=\alpha_{2}=0$. In this case, the constrained stiffness is only the stiffness which is associated with motion of the third parameter:

$$
\mathbf{K}_{\text {clamped }}=\left[\begin{array}{lll}
0 & 0 & 1
\end{array}\right] \mathbf{K}\left[\begin{array}{l}
0 \\
0 \\
1
\end{array}\right] .
$$

The constraints on a beam with clamped-pinned boundary conditions can be derived by setting (13) equal to zero and then applying the small-angle assumption. The resulting constraint is that $\alpha_{2}=3 \alpha_{1}$ :

$$
\mathbf{K}_{\text {pinned }}=\left[\begin{array}{lll}
1 & 3 & 0 \\
0 & 0 & 1
\end{array}\right] \mathbf{K}\left[\begin{array}{ll}
1 & 0 \\
3 & 0 \\
0 & 1
\end{array}\right] .
$$

Table I shows the buckling loads that are obtained by setting the determinants of (42)-(44) equal to zero. The actual Euler buckling loads, computed from [31], are shown for comparison. The Smooth Curvature model overpredicts buckling load by an amount that increases as the constraints on joint motion become more restrictive. This is to be expected; any parametric model of an elastic body will degrade in performance as the number of free parameters is reduced, because parameterization implicitly disallows any motion not captured by one of the parameterized modes. If one had a single parameter corresponding

\footnotetext{
${ }^{1}$ This approximation of constrained stiffness does not take into account the curvature of the tip constraints, if any. Nonetheless it is fairly accurate for predicting buckling load.
} 
TABLE I

CONSTRAINEd FleXURE BUCKLING LOADS

\begin{tabular}{l|c|c|c|c}
\hline \hline $\begin{array}{c}\text { Tip } \\
\text { Constraint }\end{array}$ & $\begin{array}{c}\text { Parametric } \\
\text { Constraint }\end{array}$ & $\begin{array}{c}\text { Smooth } \\
\text { Curvature } \\
\text { Buckling } \\
\text { Load }\end{array}$ & $\begin{array}{c}\text { Euler } \\
\text { Buckling } \\
\text { Load }\end{array}$ & $\begin{array}{c}\text { Percent } \\
\text { Error }\end{array}$ \\
\hline Free & - & $2.4677 \frac{E I}{L^{2}}$ & $2.4674 \frac{E I}{L^{2}}$ & $0.014 \%$ \\
\hline Guided & $\alpha_{1}=0$ & $10.0000 \frac{E I}{L^{2}}$ & $9.8696 \frac{E I}{L^{2}}$ & $1.321 \%$ \\
\hline Pinned & $\alpha_{2}=3 \alpha_{1}$ & $20.9187 \frac{E I}{L^{2}}$ & $19.7392 \frac{E I}{L^{2}}$ & $5.975 \%$ \\
\hline Clamped & $\begin{array}{c}\alpha_{1}=0 \\
\alpha_{2}=0\end{array}$ & $42.0000 \frac{E I}{L^{2}}$ & $39.4784 \frac{E I}{L^{2}}$ & $6.387 \%$ \\
\hline \hline
\end{tabular}

to each buckling mode, then it would be possible to make zeroerror predictions. The first several buckling modes of a bending beam are smooth, though, and consequently the Smooth Curvature model still produces fairly accurate results under restrictive constraints.

\section{CONCLUSION}

Nonconventional mechanisms need not be second-class citizens in robotics. In this paper, we have shown that the behavior of Euler-Bernoulli beams can be modeled in a manner that is efficient and accurate over the range of motion typically seen in a flexure joint. Unlike more general methods for elastic body modeling, such as finite element models, the Smooth Curvature model uses a minimal number of model parameters, thereby avoiding problems with poorly conditioned Jacobians. Computation of the joint kinematics is also fast, requiring only ten trigonometric function evaluations that can be reused to compute the joint Jacobian and Hessian. Furthermore, standard analyses such as predicting deformation or stiffness under load can be addressed in the familiar framework of kinematic Jacobians and Hessians and generalized stiffness matrices. These highly desirable features were obtained by avoiding ad hoc modeling methods in favor of a principled approach rooted in variational mechanics.

The model construction process employed in this paper could be applied to any number of problems: beams having nonconstant cross section, beams in torsion, bending sheets, and more abstract elastic shapes. The simplicity and elegance of the result will depend on the smoothness of the real physical system, and on the ease with which the local elastic deformation can be integrated to find the joint elastic energy and end-to-end kinematic transformation. Future work will focus on these very interesting extensions to this model.

\section{APPENDIX}

This MATLAB script provides a reference implementation of forward kinematics for the Smooth Curvature model.

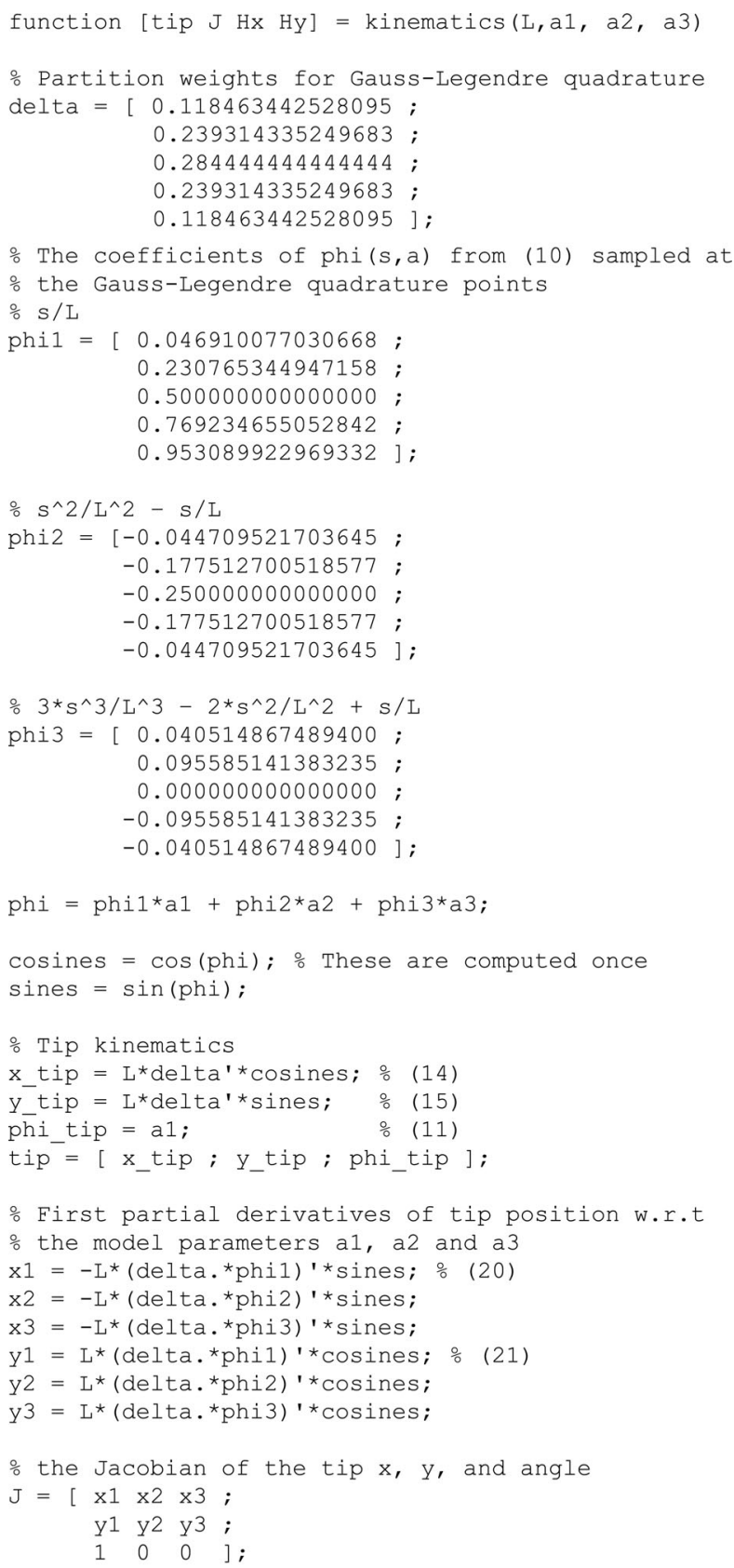

\% Second partial derivatives of the tip position $\mathrm{x} 11=-\mathrm{L}^{*}\left(\text { delta. }{ }^{*} \text { phil. }{ }^{*} \text { phil) }\right)^{\prime}{ }^{*}$ cosines; $\%$ (30) 


\section{REFERENCES}

[1] A. M. Dollar and R. D. Howe, "The highly adaptive SDM hand: Design and performance evaluation," Int. J. Robot. Res., vol. 29, pp. 585-597, 2010.

[2] F. Lotti, P. Tiezzi, G. Vassura, L. Biagiotti, G. Palli, and C. Melchiorri, "Development of UB hand 3: Early results," in Proc. IEEE Int. Conf. Robot. Autom., 2005, pp. 4499-4504.

[3] J. G. Cham, S. A. Bailey, J. E. Clark, R. J. Full, and M. R. Cutkosky, "Fast and robust: Hexapedal robots via shape deposition manufacturing," Int. J. Robot. Res., vol. 21, no. 10-11, pp. 869-882, 2002.

[4] R. J. Wood, S. Avadhanula, R. Sahai, E. Steltz, and R. S. Fearing, "Microrobot design using fiber reinforced composites," ASME J. Mech. Design, vol. 130, no. 5, p. 052304 , May 2008.

[5] A. M. Dollar and R. D. Howe, "Towards grasping in unstructured environments: Grasper compliance and configuration optimization," Adv. Robot., vol. 19 , no. 5 , pp. 523-543, 2005.

[6] R. Digiantonio and G. Lawrence, "Two-shot molding of thermoplastic elastomers," in Proc. SPE Annu. Technol. Conf., 1992, pp. 851-853.

[7] R. Merz, F. Prinz, K. Ramaswami, M. Terk, and L. Weiss, "Shape deposition manufacturing," in Proc. Solid Freeform Fabricat. Symp., 1994, vol. 5, pp. 1-8.

[8] A. Midha, T. Norton, and L. Howell, "On the nomenclature, classification, and abstractions of compliant mechanisms," Trans. ASME, vol. 116, no. 1, pp. 270-280, 1994.

[9] H. J. Su, "A pseudorigid-body 3R model for determining large deflection of cantilever beams subject to tip loads," J. Mechan. Robot., vol. 1, no. 2, p. 021008, 2009.

[10] B. Horn, "The curve of least energy," ACM Trans. Math. Softw., vol. 9, no. 4, pp. 441-460, 1983.

[11] A. Macchelli, C. Melchiorri, and S. Stramgioli, "Port-based modeling and simulation of mechanical systems with rigid and flexible links," IEEE Trans. Robot., vol. 25, no. 5, pp. 1016-1029, Oct. 2009.

[12] E. Bayo, "A finite-element approach to control the end-point motion of a single-link flexible robot," J. Robot. Syst., vol. 4, no. 1, pp. 63-75, 1986.

[13] M. Hannan and I. Walker, "Kinematics and the implementation of an elephant's trunk manipulator and other continuum style robots," J. Robot. Syst., vol. 20, no. 2, pp. 45-63, 2003.

[14] B. A. Jones and I. D. Walker, "Kinematics for multisection continuum robots," IEEE Trans. Robot., vol. 22, no. 1, pp. 43-55, Feb. 2006.

[15] S. Timoshenko and D. Young, Advanced Dynamics. New York: McGraw-Hill, 1948, pp. 205-207.

[16] A. DeLuca and B. Siciliano, "Closed-form dynamic model of planar multilink lightweight robots," IEEE Trans. Syst., Man, Cybern., vol. 21, no. 4 , pp. 826-839, Jul./Aug. 1991.

[17] L. U. Odhner and A. M. Dollar, "The smooth curvature flexure model: An accurate, low-dimensional approach for robot analysis," in Proc. Robot.: Sci. Syst. VI, 2010, pp. 137-144.

[18] L. U. Odhner and A. M. Dollar, "Fast, accurate models for predicting the compliance of elastic flexure-jointed robots," presented at the ASME Int. Design Eng. Tech. Conf. Comput., In. Eng. Conf., Montreal, QC, Canada, 2010.

[19] J. Denavit and R. S. Hartenberg, "A kinematic notation for lower-pair mechanisms based on matrices," ASME J. Appl. Mech, vol. 23, pp. 215221, 1955.

[20] R. Murray, Z. Li, and S. Sastry, A Mathematical Introduction to Robotic Manipulation. Boca Raton, FL: CRC, 1994, pp. 39-45.

[21] L. L. Howell, Compliant Mechanisms. Hoboken, NJ: WileyInterscience, 2001.
[22] G. Chirikjian and J. Burdick, "A modal approach to hyper-redundant manipulator kinematics," IEEE Trans. Robot. Autom., vol. 10, no. 3, pp. 343-354, Jun. 1994.

[23] B. Asker, "The spline curve, a smooth interpolating function used in numerical design of ship-lines," BIT Numerical Math., vol. 2, no. 2, pp. 76-82, 1962.

[24] R. L. Levien, "From spiral to spline: Optimal techniques in interactive curve design," Ph.D. dissertation, Dept. Elec. Eng. Comp. Sci., Univ. California, Berkeley, 2009.

[25] W. Press, S. Teukolsky, W. Vetterling, and B. Flannery, Numerical Recipes in C, 2nd ed. Cambridge, U.K.: Cambridge Univ. Press, 1992, pp. 190192

[26] R. Levien, "The elastica: A mathematical history," Univ. California, Berkeley, Tech. Rep. No. UCB/EECS-2008-103, 2008.

[27] H. Hanafusa and H. Asada, "Stable prehension by a robot hand with elastic fingers," in Proc. 7th Int. Symp. Ind. Robots, 1977, pp. 361-368.

[28] M. R. Cutkosky and I. Kao, "Computing and controlling compliance of a robotic hand," IEEE Trans. Robot. Autom., vol. 5, no. 2, pp. 151-165, Apr. 1989.

[29] R. W. Brockett and A. Stokes, "On the synthesis of compliant mechanisms," in Proc. IEEE Int. Conf. Robot. Autom., 1991, pp. 2168-2173.

[30] J. K. Salisbury, "Active stiffness control of a manipulator in Cartesian coordinates," in Proc. 19th IEEE Conf. Decision Control Including Symp. Adaptive Processes, 1980, Dec. 1980, vol. 19, pp. 95-100.

[31] S. Crandall, C. Dahl, and T. Lardner, An Introduction to the Mechanics of Solids, 2nd ed. New York: McGraw-Hill, 1999, p. 587.

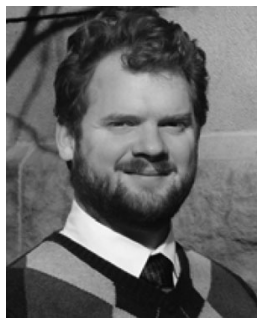

Lael U. Odhner (M'09) received the S.B., S.M., and Sc.D. degrees from the Massachusetts Institute of Technology, Cambridge.

$\mathrm{He}$ is currently a Postdoctoral Associate with Yale University, New Haven, CT. His research interests include the control of novel actuators and the design of robot arms and hands having nontraditional machine elements.

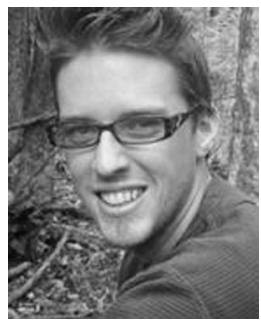

Aaron M. Dollar (M'06) received the B.S. degree in mechanical engineering from the University of Massachusetts, Amherst, and the S.M. and Ph.D. degrees in engineering sciences from Harvard University, Cambridge, MA

He is currently an Assistant Professor of Mechanical Engineering at Yale University, New Haven, CT. His research interests include robotic grasping and manipulation, tactile sensing, prosthetics and rehabilitation robotics, active exoskeletons, and robot locomotion.

Dr. Dollar is an active member of the American Society of Mechanical Engineers and the American Society of Engineering Education. He is the Editor and Cofounder of RoboticsCourseWare.org: an open repository for robotics pedagogical materials. 